\title{
Adipocytokines in SLeep ApNeA Syndrome
}

\author{
E. Wysocka ${ }^{1}$, S. Cofta ${ }^{2}$, S. Dziegielewska ${ }^{1}$, J. Gozdzik ${ }^{2}$, L. Torlinski ${ }^{1}$, H. Batura-Gabryel ${ }^{2}$ \\ ${ }^{1}$ Department of Clinical Biochemistry and Laboratory Medicine and \\ ${ }^{2}$ Department of Respiratory Medicine, Poznan University of Medical Sciences, Poznan, Poland
}

\begin{abstract}
Objective: Biomarkers of adipose tissue may affect glucose and lipid metabolism and present pro-inflammatory properties, thus could be involved in the pathobiochemistry of cardiovascular disease (CVD). The coexistence of sleep apnea syndrome (OSA) and metabolic risk factors of CVD is worth explaining. The aim of the study was to compare the serum adipocytokines in subjects with and without OSA, who had all elevated body mass index (BMI).

Methods: Overweight (BMI: 25.0-29.9 kg/m²) and obese (BMI: $30.0-39.9 \mathrm{~kg} / \mathrm{m}^{2}$ ) OSA-suspected Caucasian males, aged 30-63, with no acute disease or chronic disorder underwent polysomnographic evaluation to select OSA-positive (AHI $\geq 5$ ) and OSA-negative $(\mathrm{AHI}<5)$ subjects. Four subgroups were created of 18 persons each: Over(weight)-OSA-Neg, OverOSA-Pos, Obese-OSA-Neg, Obese-OSA-Pos. In all subjects, plasma carbohydrate and lipid metabolism parameters, and serum uric acid, resistin and leptin concentrations were determined.

Results: A decreased resistin level was observed in Over-OSA-Pos vs. Over-OSA-Neg subjects ( $\mathrm{P}=0.037)$ as well as in Obese-OSA-Pos vs. Obese-OSA-Neg $(\mathrm{P}=0.045)$. No differences in leptin concentrations were observed. A positive correlation between leptin and BMI was in both overweight subgroups and a negative one between resistin and fasting glucose was in both obese subgroups.

Conclusions: OSA may decrease the serum resistin level in subjects with excess body mass and also may contribute to glucose metabolism, but has no influence on the leptin level.
\end{abstract}

Key words: obstructive sleep apnea, body mass index, adipocytokines, leptin, resistin

\section{INTRODUCTION}

Excess body mass, overweight affecting about 34\% and obesity affecting $25-31 \%$ of population in the developed countries, increases the risk for type 2 diabetes mellitus (DM), cardiovascular disease (CVD), non-alcoholic fatty liver disease, different endocrine disorders, some forms of cancer, osteoarthropathies, and for sleep apnea [1]. Studies concerning the most common form of sleep apnea, obstructive sleep apnea (OSA), documented that $60-70 \%$ of patients are obese, hypertensive, have dyslipidemia, and $16 \%$ are diagnosed with DM [2]. Cytokines and insulin resistance are proposed to be mediators of excessive daytime sleepiness and pathogenetic factors for sleep apnea in obese patients, promoting cardiovascular disease [3]. Adipocytokines are released from the adipose tissue, including adipocytes and immune cells infiltrating fat, and affect vascular function, and immune regulation [4]. Biomarkers of adipose tissue may tell about glucose and lipid metabolism and pro-inflammatory properties; and consequently about the propensity for CVD [5].

Leptin, adipose-derived hormone, is a biomarker for body fat taking part in regulation of energy balance, including appetite and metabolism. Adipocytes are the major source of circulating leptin, but it can also be produced by skeletal muscles, fundic glands of stomach, bone marrow, ovaries, pituitary and liver [6]. The hypothalamus is the primary target for this adipocytokine, but leptin receptors are expressed also on polymorphonuclear leukocytes, monocytes, macrophages and lymphocytes. Leptin could modulate the immune response to atherosclerosis [7]. Failure of leptin production results from genetic abnormality. Regulatory defect concerns inadequately low leptin expression/secretion for a given fat mass and adipose tissue may expand in such subjects until the expected adipocytokine level is reached. Leptin resistance is associated with relative or absolute insensitivity to leptin [8]. Beside genetics, different metabolic factors are considered contributory to adipocytokine production and sensitivity [9]. Among the adipose tissue-derived signals (i.e., adiponectin, angiotensin, estradiol, IL-6, leptin, PAI-1, and TNF- $\alpha$ ), resistin and its pathophysiological role in obesity and DM are a matter of controversy. Resistin has to do with endocrine pathways involved in insulin resistance [10], and consequently could be increased in obesity [11]. It also is linked to the inflammatory response and increases the expression of the interleukins IL-1, IL- 6 , and IL-12 and of TNF- $\alpha$ [12]. Resistin is not expressed in human primary adipocytes but is present in immune cells found in white adipose tissue in obesity; adipocytes may be target cells for resistin $[13,14,15]$. There are studies showing a decreased serum concentration of resistin with increasing adiposity [16], which questions the possible role of this hormone in linking obesity to DM [17]. 
In the present study, we investigated the serum adipocytokines in overweight and obese subjects, with and without OSA.

\section{Material AND Methods}

\section{SubjeCtS AND SETTINGS}

The study was performed in accordance with the Declaration of Helsinki for Human Research, approved by the Bioethics Committee of the University of Medical Sciences in Poznan, Poland. All subjects participating in the study gave informed consent to the study procedure. Central obese non-smoking Caucasian males (at least $94 \mathrm{~cm}$ of waist circumference due to International Diabetes Federation 2005 criteria), aged 30-63, residents of Wielkopolska District in Poland, were enrolled into the study. Males with no acute disease or severe chronic disorder were screened by Epworth Sleepiness Scale. OSA suspected persons were evaluated in the Sleep Laboratory of the Department of Respiratory of Medicine of the University of Medical Sciences in Poznan, Poland. All studied persons were qualified for oral glucose tolerance test (OGTT) due to WHO recommendations. Newly diagnosed DM subjects were excluded from the study. Due to known gender differences, only male subjects were included. BMI, blood pressure, and metabolic factors were analyzed regarding the influence of OSA on the adipocytokine concentrations in the blood. Subjects were stratified into groups, 18 persons each, according to the BMI: overweight (Over) 25.0-29 and obese (Obese) 30.0-34.9 $\mathrm{kg} / \mathrm{m}^{2}$. Apnea/hypopnea index $(\mathrm{AHI} \geq 5)$ was used to diagnose OSA-positive (OSA-Pos) and OSA-negative (OSA-Neg) (AHI <5). Accordingly, there were 4 groups: Over-OSA-Pos and Over-OSA-Neg, and Obese-OSA-Pos and Obese-OSA-Neg.

\section{MEASUREMENTS}

Complete physical examination, systolic (SBP) and diastolic blood pressure (DBP) were taken. OSA-suspected persons underwent full-night polysomnography, using EMBLA S4000 system (Remlogic; EMBLA Co., Denver, Colorado). Airflow was monitored by a nasal flow cannula. Abdominal and thoracic movements were assessed by respiratory inductive plethysmography. Night recordings of arterial oxygen saturation were obtained by finger pulsoximetry. Snoring sounds, heart rate, and sleep position also were recorded. Apnea was defined as a cessation of airflow lasting more than $10 \mathrm{~s}$, and hypopnea was defined as a discrete reduction (two thirds) of airflow and/or abdominal ribcage movements lasting more than $10 \mathrm{~s}$ and associated with a decrease of more than $4 \%$ in oxygen saturation.

Fasting glucose (G 0), glucose at $120 \mathrm{~min}$ (G 120) of the OGT'T, total cholesterol (T-C), HDL cholesterol (HDL-C), triglycerides (TG), uric acid (UA) concentrations, and plasma glucose at G 120 were determined using enzymatic methods (BioMérieux, Marcyl'Etoile, France) and UV-160A Shimadzu spectrophotometer (Shimadzu, Kyoto, Japan). LDL cholesterol (LDL-C) was calculated from Friedewald's formula. Fasting serum insulin (Ins 0) was measured by ELISA method (BioSource, Nivelles, Belgium) on microplate reader Sunrise ${ }^{T M}$ (Tecan Group, Männedorf, Switzerland). Insulin resistance (IR) was calculated as Ins/G0 ratio. Homeostasis model assessment of insulin resistance (HOMA-IR), defined as Ins $0 \times \mathrm{xG} 0 / 22.5$, was established.

Serum samples were stored at $-80^{\circ} \mathrm{C}$ until leptin and resistin were measured using ELISA (R\&D Systems, Minneapolis, Minnesota) on a microplate reader Sunrise $^{\text {TM }}$ (Tecan Group, Männedorf, Switzerland). The minimum detectable levels of leptin and resistin were $0.0078 \mathrm{ng} / \mathrm{ml}$ and $0.026 \mathrm{ng} / \mathrm{ml}$, respectively. The intraassay coefficients of variation (CV) for leptin (2.5$3.0 \%$ ) and resistin (5.1-5.3\%) and the inter-assay CV for leptin $(4.7-5.4 \%)$ and resistin $(6.5-7.0 \%)$ were calculated.

\section{STATISTICAL ANALYSIS}

All results are shown as means $\pm \mathrm{SD}$ and medians. The normality of value distributions was checked by Shapiro-Wilk's test. Significant differences between studied groups were calculated by nonparametric Man Whitney-U test and correlations were described by Spearman's coefficient $(\mathrm{R})$, while $\mathrm{P}<0.05$. Data were analyzed with Statistica version 6.0 packet.

\section{RESULTS}

Clinical and laboratory data of the studied groups are shown in Table 1. The Over-OSA-Pos and Over-OSANeg as well as Obese-OSA-Pos and Obese-OSA-Neg groups did not differ regarding the age, BMI, SBP and DBP, glycemia during OGTT, insulinemia, and plasma lipid and uric acid concentrations.

A decreased level of resistin was found in both Over-OSA-Pos and Obese-OSA-Pos subjects compared with the respective Neg groups (Fig. 1). No differences in the leptin concentration were observed in both BMI categories.

A positive correlation between leptin and BMI was found in both overweight groups of subjects, OverOSA-Pos and Over-OSA-Neg $(r=0.60$ and $r=0.44$, respectively; $\mathrm{P}<0.05$ ), and a negative correlation between the levels of resistin and $G 0$ in both obese

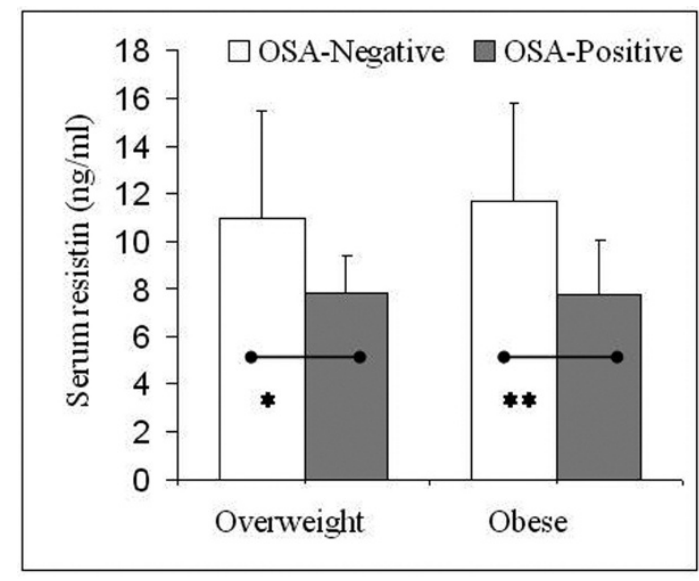

Fig. 1. Comparison of serum resistin concentration between the studied groups; $* \mathrm{P}=0.037, * * \mathrm{P}=0.045$. 
Table 1. Clinical and biochemical characteristics of the studied groups.

\begin{tabular}{|c|c|c|c|c|c|c|c|c|}
\hline & \multicolumn{2}{|c|}{ Over-OSA-Neg } & \multicolumn{2}{|c|}{ Over-OSA-Pos } & \multicolumn{2}{|c|}{ Obese-OSA-Neg } & \multicolumn{2}{|c|}{ Obese-OSA-Pos } \\
\hline & Mean \pm SD & Median & Mean \pm SD & Median & Mean \pm SD & Median & Mean \pm SD & Median \\
\hline Age (years) & $50 \pm 11$ & 53 & $52 \pm 10$ & 53 & $51 \pm 8$ & 53 & $50 \pm 8$ & 51 \\
\hline AHI (\#/h) & $2.4 \pm 0.8$ & 2.5 & $31.0 \pm 20.0$ & 36.0 & $1.8 \pm 1.5$ & 2.0 & $36.0 \pm 23.5$ & 35.8 \\
\hline BMI $\left(\mathrm{kg} / \mathrm{m}^{2}\right)$ & $28.3 \pm 1.7$ & 28.2 & $28.1 \pm 2.0$ & 27.7 & $34.3 \pm 2.4$ & 34.4 & $33.6 \pm 2.8$ & 32.9 \\
\hline $\mathrm{SBP}(\mathrm{mmHg})$ & $130 \pm 19$ & 127 & $130 \pm 10$ & 130 & $137 \pm 10$ & 135 & $142 \pm 18$ & 140 \\
\hline DBP (mmHg) & $85 \pm 12$ & 82 & $81 \pm 7$ & 80 & $90 \pm 8$ & 90 & $86 \pm 13$ & 80 \\
\hline G $0(\mathrm{mmol} / \mathrm{l})$ & $5.48 \pm 0.82$ & 5.17 & $5.45 \pm 0.37$ & 5.49 & $5.54 \pm 0.69$ & 5.66 & $5.45 \pm 0.55$ & 5.45 \\
\hline $\mathrm{G} 120(\mathrm{mmol} / \mathrm{l})$ & $6.50 \pm 1.60$ & 6.50 & $6.40 \pm 1.76$ & 6.64 & $6.43 \pm 1.63$ & 6.41 & $6.24 \pm 1.68$ & 5.89 \\
\hline Ins $0(\mathrm{mU} / \mathrm{l})$ & $21.7 \pm 15.0$ & 19.7 & $24.4 \pm 5.2$ & 23.7 & $22.1 \pm 6.7$ & 19.2 & $24.4 \pm 10.1$ & 19.8 \\
\hline IR & $0.27 \pm 0.19$ & 0.21 & $0.24 \pm 0.05$ & 0.23 & $0.23 \pm 0.07$ & 0.21 & $0.28 \pm 0.10$ & 0.246 \\
\hline HOMA-IR & $6.2 \pm 4.0$ & 5.8 & $6.1 \pm 1.5$ & 6.1 & $5.4 \pm 1.9$ & 4.7 & $6.5 \pm 3.1$ & 5.9 \\
\hline $\begin{array}{l}\text { OGTT: } \\
\text { normal }\end{array}$ & $\mathrm{n}=10(56 \%)$ & & $\mathrm{n}=9(50 \%)$ & & $\mathrm{n}=7(39 \%)$ & & $\mathrm{n}=7(39 \%)$ & \\
\hline preDM & $\mathrm{n}=8 \quad(44 \%)$ & & $\mathrm{n}=9(50 \%)$ & & $\mathrm{n}=11(61 \%)$ & & $\mathrm{n}=11(61 \%)$ & \\
\hline $\mathrm{T}-\mathrm{C}(\mathrm{mmol} / \mathrm{l})$ & $6.05 \pm 1.46$ & 5.97 & $5.90 \pm 1.19$ & 5.94 & $5.48 \pm 1.17$ & 5.43 & $5.47 \pm 1.07$ & 5.82 \\
\hline TAG (mmol/l) & $1.64 \pm 0.97$ & 1.74 & $1.95 \pm 1.05$ & 1.67 & $2.33 \pm 1.12$ & 2.20 & $1.89 \pm 1.10$ & 1.48 \\
\hline HDL-C (mmol/l) & $1.22 \pm 0.34$ & 1.25 & $1.19 \pm 0.22$ & 1.16 & $1.17 \pm 0.30$ & 1.16 & $1.10 \pm 0.16$ & 1.12 \\
\hline LDL-C (mmol/l) & $4.07 \pm 1.32$ & 3.80 & $3.87 \pm 1.00$ & 3.88 & $3.35 \pm 0.95$ & 3.32 & $3.62 \pm 0.97$ & 3.83 \\
\hline $\mathrm{UA}(\mu \mathrm{mol} / \mathrm{l})$ & $331 \pm 91$ & 332 & $326 \pm 59$ & 338 & $382 \pm 105$ & 350 & $359 \pm 70$ & 371 \\
\hline Resistin $(\mathrm{ng} / \mathrm{ml})$ & $11.00 \pm 4.44$ & 11.07 & $7.80 \pm 1.60$ & 7.90 & $11.70 \pm 4.10$ & 11.49 & $7.75 \pm 2.29$ & 7.17 \\
\hline Leptin (ng/ml) & $21.74 \pm 15.00$ & 19.66 & $21.31 \pm 14.95$ & 18.61 & $22.86 \pm 9.01$ & 22.03 & $22.67 \pm 13.42$ & 19.56 \\
\hline
\end{tabular}

groups of subjects, Obese-OSA-Pos and Obese-OSANeg $(r=-0.63$ and $r=-0.54$, respectively; $\mathrm{P}<0.05)$.

\section{DISCUSSION}

There is an explosion in research and clinical interest in bidirectional association between sleep apnea and metabolic abnormalities. Sleep fragmentation and intermittent hypoxemia are proposed to implicate sleep apnea as an independent risk factor for metabolic abnormalities [18]. Shortened sleep duration was demonstrated to provoke insulin resistance and glucose intolerance [19].

The coexistence of obesity and OSA is well known. Adiposity-related signals such as insulin and leptin circulate in proportion to body fat composition and should control satiety balance [20]. Resistin, adiposityrelated but immune cell-derived hormone is considered as one possible proinflammatory factor that may link obesity-related metabolic disturbances to CVD.

Previous studies show that increased serum leptin levels in OSA subjects correlate with BMI, insulin resistance, and high incidents of CVD [21, 22]. A Japanese study found elevated leptin concentrations with increasing severity of OSA, while the subjects with severe OSA presented higher BMI and insulin resistance than those with moderate OSA [23]. These authors did not find any differences in the leptin level between moderate OSA and control subjects. The findings suggest that the degree of obesity may be a more important factor for elevated leptin level in OSA patients than the severity of disease.

The present study adds to the controversy surrounding the level of leptin in subjects with increased body mass, OSA subjects in particular, supporting the role of adiposity-derived proinflammatory state, rather than obesity per se, in the pathogenesis of CVD. That is why recently we refer to discuss 'metabolically healthy but obese' individuals in the context of cardiovascular risk factors [24]. It is also suggested that leptin receptor gene polymorphism could be associated with the prevalence of OSA, and might determine obesity and lipid disturbances in homozygotic Arg/Arg genotype carries [25].

The present study on overweight and obese subjects shows no differences in their carbohydrate and lipid metabolic parameters as well as insulin resistance. Both OSA positive and negative subjects had similar leptin concentrations and, rather surprisingly, decreased serum resistin levels in both overweight and obese OSA positive subjects negatively correlated with the fasting glucose level. Positive correlations between resistin, insulin resistance, and carbohydrate disturbances have recently been reported and explained mainly by engaged proinflammatory state [10]. Controversies concerning resistin can be found in other reports on the subject. Resistin levels do not change during effective CPAP therapy, whereas insulin sensitivity index improves in the OSA patients which also closely relates with the inflammatory parameters [26]. In that study, resisitin concentration increased in OSA patients who lost weight and decreased in those who gained weight [26]. Elevated serum levels of resistin and IL- 6 have been found in OSA male subjects compared with those without OSA [27], which suggests a relationship between and IL-6 changes. Since the latter is a marker of inflammatory state, inflammation-asso- 
ciated serum resistin changes in OSA patients are suggested. In the study in question [27], leptin level did not change in OSA subjects, which is in accord with the present results.

We conclude that serum resistin is decreased in obstructive sleep apnea subjects of excess body mass, whereas serum leptin level is unaffected.

Acknowledgements: The research was supported by Poznan University of Medical Sciences, Poland.

Conflict of interest: The authors declared no conflicts of interest in relation to this work.

\section{REFERENCES}

[1] Gurevich-Panigrahi T, Panigrahi S, Wiechec E, Los M. Obesity: pathophysiology and clinical management. Curr Med Chem 2009; 16(4): 506-21.

[2] Kiely JL, McNicholas WT. Cardiovascular risk factors in patients with obstructive sleep apnoea syndrome. Eur Respir J 2000; 16(1): 128-33.

[3] Vgontzas AN, Bixlera EO, Chrousos GP. Sleep apnea is a manifestation of the metabolic syndrome. Sleep Med Rev 2005; 9(3): 211-24.

[4] Guzik TJ, Mangalat D, Korbut R. Adipocytokines - novel link between inflammation and vascular function? J Physiol Pharmacol 2006; 57(4): 505-28.

[5] Rondinone CM. Adipocyte-derived hormones, cytokines, and mediators. Endocrine 2006; 29(1): 81-90.

[6] Brennan AM, Mantzoros CS. Drug Insight: the role of leptin in human physiology and pathophysiology - emerging clinical applications. Nat Clin Pract Endocrinol Metab 2006; 2(6): 318-27.

[7] Taleb S, Herbin O, Ait-Oufella H, Verreth W, Gourdy P, Barateau V, Merval R, Esposito B, Clement K, Holvoet P, Tedgui A, Mallat Z. Defective leptin/leptin receptor signaling improves regulatory $T$ cell immune response and protects mice from atherosclerosis. Arterioscler Thromb Vasc Biol 2007; 27(12): 2691-8.

[8] Margetic S, Gazzola C, Pegg GG, Hill RA. Leptin: a review of its peripheral actions and interactions. Int J Obes Relat Metab Disord 2002; 26(11): 1407-33.

[9] Schwartz MW, Porte D Jr. Diabetes, obesity, and the brain. Science 2005; 307(5708): 375-9.

[10] Silha JV, Krsek M, Skrha JV, Sucharda P, Nyomba BL, Murphy LJ. Plasma resistin, adiponectin and leptin levels in lean and obese subjects: correlations with insulin resistance. Eur J Endocrinol 2003; 149(4): 331-5.

[11] Degawa-Yamauchi M, Bovenkerk JE, Juliar BE, Watson W, Kerr K, Jones R, Zhu Q, Considine RV. Serum resistin (FIZZ3) protein is increased in obese humans. J Clin Endocrinol Metab 2003; 88(11): 5452-5.

[12] Silswal N, Singh AK, Aruna B, Mukhopadhyay S, Ghosh S, Ehtesham NZ. Human resistin stimulates the pro-inflammatory cytokines TNF-alpha and IL-12 in macrophages by NF-kappaB-dependent pathway. Biochem Biophys Res Commun 2005; 334(4): 1092-101.

[13] Cinti S, Mitchell G, Barbatelli G, Murano I, Ceresi E, Faloia E, Wang S, Fortier M, Greenberg AS, Obin MS. Adipocyte death defines macrophage localization and function in adipose tissue of obese mice and humans. J Lipid Res 2005; 46(11): 2347-55.

[14] Curat CA, Wegner V, Sengenes C, Miranville A, Tonus C, Busse R, Bouloumie A. Macrophages in human visceral adipose tissue: increased accumulation in obesity and a source of resistin and visfatin. Diabetologia 2006; 49(4): 744-7.
[15] Vendrell J, Broch M, Vilarrasa N, Molina A, Gómez JM, Gutiérrez C, Simón I, Soler J, Richart C. Resistin, adiponectin, ghrelin, leptin, and proinflammatory cytokines: relationships in obesity. Obes Res 2004; 12(6): $962-71$.

[16] Lee JH, Chan JL, Yiannakouris N, Kontogianni M, Estrada E, Seip R, Orlova C, Mantzoros CS. Circulating resistin levels are not associated with obesity or insulin resistance in humans and are not regulated by fasting or leptin administration: cross-sectional and interventional studies in normal, insulin-resistant, and diabetic subjects. J Clin Endocrinol Metab 2003; 88(10): 4848-56.

[17] Heilbronn LK, Rood J, Janderova L, Albu JB, Kelley DE, Ravussin E, Smith SR. Relationship between serum resistin concentrations and insulin resistance in nonobese, obese, and obese diabetic subjects. J Clin Endocrinol Metab 2004; 89(4): 1844-8.

[18] Aurora RN, Punjabi NM. Sleep apnea and metabolic dysfunction: cause or co-relation? Sleep Med Clin 2007; 2(2): 237-50.

[19] Spiegel K, Knutson K, Leproult R, Tasali E, Van Cauter E. Sleep loss: a novel risk factor for insulin resistance and type 2 diabetes. J Appl Physiol 2005; 99(5): 2008-19.

[20] Morton GJ, Blevins JE, Williams DL, Niswender KD, Gelling RW, Rhodes CJ, Baskin DG, Schwartz MW. Leptin action in the forebrain regulates the hindbrain response to satiety signals. J Clin Invest 2005; 115(3): 70310.

[21] Ip MS, Lam KS, Ho C, Tsang KW, Lam W. Serum leptin and vascular risk factors in obstructive sleep apnea. Chest 2000; 118(3): 580-6.

[22] Schäfer H, Pauleit D, Sudhop T, Gouni-Berthold I, Ewiq S, Berthold HK. Body fat distribution, serum leptin, and cardiovascular risk factors in men with obstructive sleep apnea. Chest 2002; 122(3): 829-39.

[23] Tokuda F, Sando Y, Matsui H, Koike H, Yokoyama T. Serum levels of adipocytokines, adiponectin and leptin, in patients with obstructive sleep apnea syndrome. Inter Med 2008; 47(21): 1843-9.

[24] Karelis AD, Brochu M, Rabasa-Lhoret B. Can we identify metabolically healthy but obese individuals (MHO)? Diabetes Metab 2004; 30(6): 569-72.

[25] Popko K, Gorska E, W_sik M, Stoklosa A, P_ywaczewski R, Winiarska M, Gorecka D, Sliwinski P, Demkow U. Frequency of distribution of leptin receptor gene polymorphism in obstructive sleep apnea patients. J Physiol Pharmacol 2007; 58 Suppl 5: 551-61.

[26] Harsch IA, Koebnick C, Wallaschofski H, Schahin SP, Hahn EG, Ficker JH, Lohmann T, Konturek PC. Resistin levels in patients with obstructive sleep apnoea syndrome - the link to subclinical inflammation? Med Sci Monit 2004; 10(9): CR510-5.

[27] Yamamoto Y, Fuijuchi S, Hiramatsu M, Nishigaki Y, Takeda A, Fujita Y, Yamazaki Y. Resistin is closely related to systemic inflammation in obstructive sleep apnea. Respiration 2008; 76(4): 377-85.

\section{Corresponding author:}

Dr med. Ewa Wysocka

Department of Clinical Biochemistry and Laboratory

Medicine

Poznan University of Medical Sciences

Grunwaldzka 6 St.

60-780 Poznan

Poland

Phone: +48618546590

Fax: $\quad+48618546599$

E-mail: ewysocka@ump.edu.pl 